\title{
Role of cystitis glandularis and intestinal metaplasia in the development of a bladder benign polypoid tumor in a pediatric patient (case report)
}

\begin{abstract}
Maria Popescu, Gabriel Dragan
Department of Pediatric Surgery, "Victor Gomoiu" Clinical Hospital for Children, Bucharest, Romania

ABSTRACT

Benign tumoral bladder tumors in children are extremely rare. The inflammatory condition determined by the presence of glandular cystitis and intestinal metaplasia, coexisting or separately, can be a determinant factor for the development of these bladder masses. Both entities have been suggested to represent a precursor for bladder adenocarcinoma. We present the case of an autistic adolescent that presented with a polypoid bladder mass and urinary tract infection. We performed a cystoscopic examination and biopsy of the tumor and due to the large dimensions and preliminary histological results we decided to perform a laparotomy and excision of the mass and bladder wall that was attached to. Post operatory evolution and follow-up showed no recurrence or progression of the dysplasia towards adenocarcinoma.
\end{abstract}

Keywords: glandular cystitis, intestinal metaplasia, benign tumor, adenocarcinoma

\begin{abstract}
Abbreviations
$\mathrm{CG}$ - cystitis glandularis

IM - intestinal metaplasia

UTI - urinary tract infection
\end{abstract}

\section{INTRODUCTION}

Urinary bladder tumors in children are rare. The histological findings and the case prognosis for the pediatric population are very different from those in adults (1). The most common one, rhabdomyosarcoma has a maximum reported incidence of 4 cases per 1 million children and the benign bladder lesions are even less frequent. Papillary urothelial neoplasm, hemangiomas, neurofibromas, leiomyomas are the most common benign processes of the bladder and are hard to differentiate from masses derived from more frequent pathologies such as cystitis glandularis (CG) and intestinal metaplasia (IM) that associate with chronic inflammation and infection and tend to usually exist as multiple lesions (2). Terms like pseudotumoral cystitis or "cystitis glandularis of intestinal type" are found in the scientific literature and describe mass like lesions of the bladder wall that have an inflammatory background $(1,2)$. IM can be found alone or associated with $\mathrm{CG}$ and can be identified in normal bladder mucosa, inflammatory pathologies or even in carcinomas (3). Due to some sporadic cases that associate $\mathrm{CG}$ and IM with bladder adenocarcinoma, prompted for these two conditions to be considered as premalignant by several studies that recommend bladder biopsies and annual cystoscopic surveillance (4).

We report the case of a 14-year-old girl who was diagnosed with a bladder mass that proved to be a polypoid lesion determined by the coexistence of CG and IM. We received the informed consent of the parents to publish the data, the clinical and imaging findings of the patient. 


\section{CASE REPORT}

\section{Presenting concerns}

We present the case of a 14-year-old autistic girl that was admitted to our surgical department to investigate the suspicion of a bladder mass. The patient is non-verbal, hypersensitive to touch and very agitated. We were able to obtain the patient history from the mother, that described an increase in the level of agitation in the last 48 hours, associated with frequent urination and incontinence. The patient received antibiotic treatment (amoxicillin/ clavulanate potassium and sulfamethoxazole) in the last 6 months for two episodes of urinary tract infections (UTI). She had no other medical background and did not receive any specific medication for the autism spectrum disorder.

\section{Clinical findings and diagnostic focus}

The initial clinical examination of the patient was impossible to perform due to extreme agitation. We were able to obtain some abdominal ultrasound images that revealed the presence of a $5 / 4$ $\mathrm{cm}$ bladder mass that occupied almost the entire cavity (Fig. 1). We decided to perform a CT scan under general anesthesia that measured a $6 / 4 / 5 \mathrm{~cm}$ tumor arising from the anterior wall of the bladder and protruding inside the cavity (Fig. 2, 3). The radiologist raised the suspicion of a rhabdomyosarcoma of the anterior bladder wall. No other pathologic abdominal findings were seen on the CT scan. During the general anesthesia we were also able to palpate the abdomen (no palpable mass), to examine the genital area that presented signs of chronic inflammation and to obtain sterile urinary samples for the lab work that revealed the presence of a urinary tract infection (E Coli). Other laboratory data showed no abnormalities.

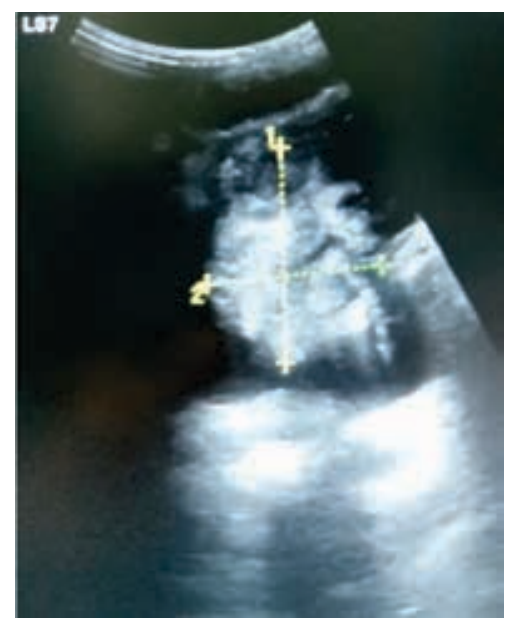

FIGURE 1. Transabdominal ultrasound of the bladder showing a large bladder mass

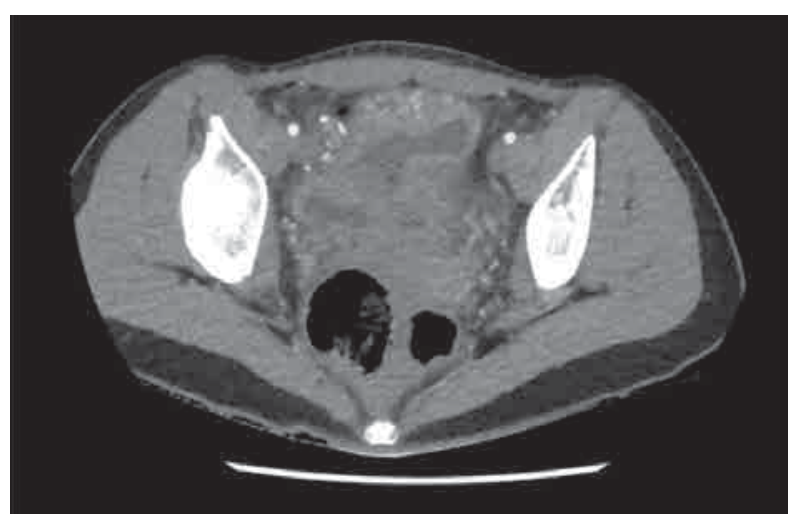

FIGURE 2. Bladder mass, CT scan (transversal view)

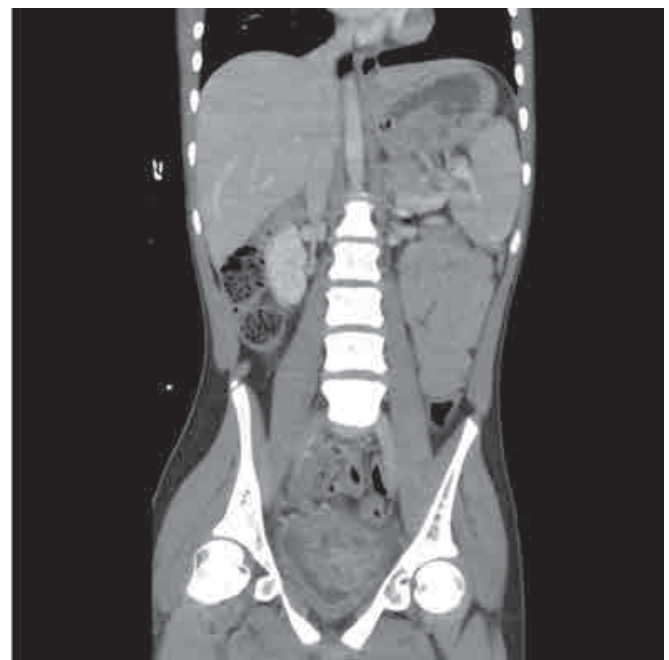

FIGURE 3. Bladder mass, CT scan (anterior view)

\section{Therapeutic focus and assessment}

We started the antibiotic treatment for the UTI and following further discussion with the parents, a decision was made to proceed to cystoscopy under general anesthesia for evaluation and possible resection or biopsy of the lesion. On cystoscopic examination we identified one large polypoid lesion that apparently was not attached to any of the bladder walls, friable and with intense vascularization (Fig. 4). Due to the large dimensions of the tumor, we decided not to resect it and we performed a biopsy. The patient was discharged 24 hours after the procedure and received antibiotic treatment at home waiting for the biopsy results. The histological examination of the tissue revealed IM and GC but could not exclude the presence of malignant cells in other parts of the tumor.

We decided to perform a laparotomy procedure, using a Pfannenstiel incision, and removed the bladder mass $(6 / 5 / 4 \mathrm{~cm})$ that was attached to the dome by a thin vascular cord (Fig. 5). We resected also the bladder wall $1 \mathrm{~cm}$ around the insertion of the cord. A Foley catheter was left in place for the bladder drainage. No other pathological findings were identified. 


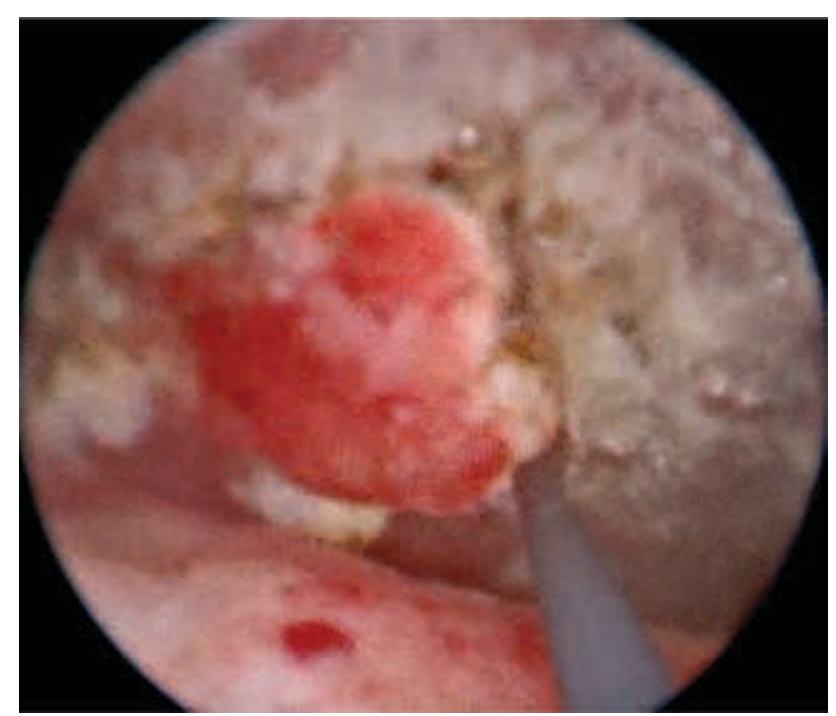

FIGURE 4. Cystoscopic view of the bladder tumor

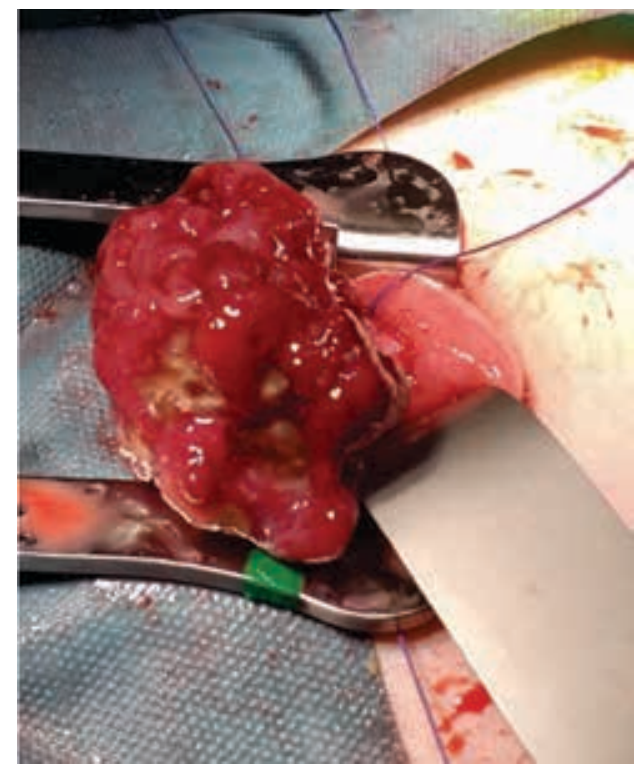

FIGURE 5. Intraoperatory aspect of the tumor

\section{Follow-up and monitoring}

24 hours after the surgical procedure the patient presented with recurrent high fever, abdominal pain, vomiting and diarrhea. The stool exam revealed a Clostridium A and B infection and we started the patient on oral Vancomycin. We removed the Foley catheter 4 days after surgery due to extreme agitation and discomfort. The patient was discharged, with the obligation of continuing the antibiotic therapy at home for another 10 days. Follow up after 14 days revealed no complication. The histopathological result revealed a polypoid tumor with areas of GC and IM with no evidence of dysplasia or malignancy.

We performed a follow up cystoscopy in six months' time, with no pathological findings. Monthly urinary tests did not show any signs of UTI.

\section{DISCUSSION}

The IM can occur alone or in association with GC. The most frequently it affects the bladder trigone, followed by the dome localization, posterior wall and lateral wall. Rarely affects children and is characterized by the presence of intestinal type of epithelium $(1,5)$. The GC can coexist with the IM, as was the case of our patient, that had a rare localization of the tumor, mostly inside the cavity but also attached to the bladder dome. She had one major risk factor that can lead to the diagnosis of GC and IM, the presence of recurrent UTI. Other associations described in the literature are with prior bladder exstrophy repair, pelvic lipomatosis, bladder stones and neurogenic bladder $(2,6)$.

Both GC and IM can present with abdominal suprapubic pain, acute urinary retention, incontinence, urinary frequency and urgency, possible hematuria $(2,7)$. Our patient suffers from autistic spectrum disorder making the clinical examination almost impossible due to extreme agitation and hyper sensibility to touch. We had to rely on general anesthesia in order to collect imagistic data that can orient a diagnosis and help establish a therapeutic management plan.

The ultrasound is the first line imaging that we use to asses bladder lesions in children but the accuracy of the result is influenced by many clinical factors (distention of the bladder, agitation of the child so). To compete the imaging diagnosis, we can perform an MRI that might be helpful when a small bladder lesion is difficult do differentiate or a CT scan as we performed for our patient that needed a reduced general anesthesia time and also assessment for possible intraabdominal metastatic lesions. There are no standardized protocols for long term follow up after a bladder tumor excision in children. We recommend ultrasound to be performed every 3 months and after 6 months a cystoscopic examination. In theory the epithelium may return to normal pattern if the stimulus that caused the GC and the IM is removed or ceased $(2,7)$. The follow-up assures that we have no recurrence and that no progression of the dysplasia was made towards a subsequent adenocarcinoma.

The therapeutic management of the bladder lesions consists in transurethral resection or partial cystectomy that can be performed for flat lesions or polypoid masses followed by long term antibiotic prophylaxis for UTI, cystoscopy every 6 months and close follow-up of the symptoms $(2,5)$. 


\section{CONCLUSIONS}

We reported a rare case of a bladder polypoid mass developed due to the coexistence of the GC and IM. The main risk factor was the presence of detected and undetected UTI and the timing of the clinical discovery was influenced by the lack of communication of the patient with the family and

Conflict of interest: none declared

Financial support: none declared

\section{REFERENCES}

1. Smith AK, Hansel DE, Jones JS, et al. Role of cystitis cystica et glandularis and intestinal metaplasia in development of bladder carcinoma. Urology. 2008;71:915-918.

2. Susan C, Armando J, Gupta A, et al. Pearls and Pitfalls in Diagnosing Pediatric Urinary Bladder Masses. RadioGraphics. 2017; 37:1872-1891.

3. Garcia D, Prera A, Saez A, et al. Transformation of glandular cystitis into bladder transitional carcinoma with adenocarcinoma areas. Arch Esp Urol.1997;50:187-189.

4. Heyns CF, De Kock ML, Kirsten PH, et al. Pelvic lipomatosis associated with cystitis glandularis and adenocarcinoma of the bladder. J Urol.1991;145:364-366. doctors due to the autistic disorder. The laparotomy procedure was chosen as a method of treatment because of the large dimensions of the tumor and the biopsy preliminary findings. The follow-up will consist in periodic cystoscopies and imaging procedures as recurrence risk and premalignant potential cannot be completely excluded.

5. Huppmann AR, Pawel BR. Polyps and masses of the pediatric urinary bladder: a 21-year pathology review. Pediatr Dev Pathol. 2011;14:438-444.

6. Capozza N, Collura G, Nappo S, et al. Cystitis glandularis in children. BJU Int. 2005;95:411-413.

7. Defoor W, Minevich E, Sheldon C. Unusual bladder masses in children. Urology. 2002;60:911-915. 